\title{
A QOS-SENSITIVE MAC FOR SLOTTED WDM METROPOLITAN RINGS
}

\author{
J.D. Angelopolous, N. Leligou, H. Linardakis, A. Stavdas \\ National Technical University of Athens
}

\begin{abstract}
Traffic segregation is essential in shared medium systems to allow best-effort traffic to coexist with delay-sensitive traffic without the former disturbing the QoS of the latter. Rigid allocation per traffic class is very inefficient so a dynamic method that exploits the elastic behavior of best-effort traffic due to its closed loop control is essential for high system utilization. A novel MAC mechanism for slotted WDM rings based on class reservations is presented and evaluated in this paper. It allows the reservations to fluctuate in accordance with the demand for real-time traffic leaving the rest of the bandwidth for besteffort traffic. Thus the QoS-demanding traffic experiences no competition from best-effort traffic while the latter is inserted whenever spare bandwidth exists to increase system efficiency.
\end{abstract}

Key words: WDM, metropolitan rings, MAC protocol, access arbitration, QoS, access priorities

\section{INTRODUCTION}

The technology of dense WDM plays an ever-increasing role in networking as new developments come to improve existing components and introduce new solutions. As traffic volumes continue to more than double each year, optical solutions seem to be spreading into territory once dominated by electrical systems. It has long ago become clear that the potential of WDM technology goes far beyond multiplying the bandwidth capacity in the transmission plant [1], [2]. Still some co-existence with electrical components is indispensable and the appropriate choice of balance is the key to cost-effectiveness. The dominance of the IP traffic strongly motivates the pursuit of optical packet switched solutions despite the 
difficulty of fast control in the context of optical networks which seem to still favour circuit-switching. However advances in burst mode components make fast packet switching feasible for the payload, even if still the control has to remain in the electronic domain [2].

Although best-effort traffic dominates traditional IP networks, novel optics-based designs for terabit networks cannot ignore the need to support traffic with demanding QoS. Any further strong increase in traffic that may help to make optical packet switching economically viable can only come from widespread deployment of audio and mainly video-intensive services.

With the above considerations in mind, the DAVID consortium chose a WDM-based packet-switching architecture as the basis for its system. It consists of a number of unidirectional slotted multi-wavelength rings of metropolitan dimensions, which collect traffic from several optical nodes. The nodes interface to edge routers/switches at the end of access networks via a variety of $1 / F s$ (e.g. Gigabit Ethernet in business areas, PONs in mixed or residential areas, cable head-ends, or any other legacy system). In the full architecture the rings are interconnected via bufferless hubs to other rings and to a mesh of packet-switched optical Add/Drop Multiplexers in the core creating a complete optical WAN. The optical packet duration $(1 \mu \mathrm{s})$ and format are fixed for all kinds of encapsulated traffic to allow for easy burst operation and optical switching. In the rest of this work the scope will be limited to the operation of a single ring focusing on a novel MAC mechanism featuring prioritised access to the slotted WDM ring so as to support QoS-intensive services.

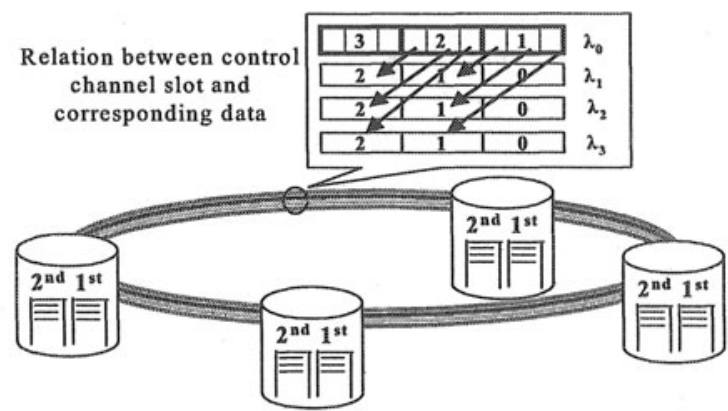

Figure 1. Control and data channel 


\section{THE CONCEPT OF MAC OPERATION IN THE METRO RING}

Each node is assumed to be equipped with a set of fixed transmitters and receivers equal in number to the wavelengths. If a smaller number, or just one, tunable transmitter/receiver is used, the problem of head of line blocking will reduce performance unless virtual output queuing algorithms are employed. The latter have been extensively studied in the context of input queued switch architectures (but also WDM rings [3]). Similar results are obtained in this ring so there is much new in this respect and this issue will not be considered here using the limited space to focus on the novel QoS priority mechanism.

Up to 32 wavelengths running at $10 \mathrm{Gbps}$ can be available on each ring with a slot size of 10000 bits $(1 \mu \mathrm{s})$. The slots on all wavelengths are synchronised therefore creating multi-lambda slots accessed on the basis of control information carried out for all wavelengths in corresponding fields of a control channel is the way illustrated in Figure 1, which shows how control fields are related to data slots. One wavelength is devoted exclusively to this control channel, which runs at $2.5 \mathrm{Gbps}$ therefore each control slot contains 2500 bits. The ring is a shared medium requiring a Medium Access Control (MAC) protocol to arbitrate access to its slots regulating both the time and wavelength dimensions. So the overall system works as a combined WDMA/TDMA distributed multiplexer.

Data slots contain payload only and the nodes are not allowed to alter them unless they are addressed to them. All addressing and control information for each data slot resides in the corresponding control slot with a one-to one correspondence. Thus, packets are not changed from optical media, buffered, re-formatted or processed inside the ring, except at the edge routers.

The format of the control channel is depicted in figure 2. The $S$ bit indicates whether the corresponding data slot is empty (0) or carries payload (1). The P bit indicates the priority of the current payload (I is for high priority). The $\mathrm{R}$ bit is used to reserve the corresponding slot while SA and DA are the source and destination addresses of the corresponding data slot in the corresponding $\lambda$ channel. The slots in the control channel have a locked timing relationship to the data multi-slots but they arrive earlier in each node by a fixed amount of time to give time for processing the contents of the control slot (and tuning time in case of tunable tranceivers) before the relevant data slot has left the current node. Only the information of the control channel is converted to electrical for processing in transit nodes while all the bulk of user information remains, until its final destination in 
the end ring, in optical form simply delayed by the fixed amount of time ( 2 slot times) until control is processed.

Each station that has packets to transmit must search for an empty slot in any $\lambda$. This is evident by the fact that the $S$ bit (slot Status) is reset. The station grabs the slot by setting this bit and inserting the destination address in the relevant DA field. Nodes can only write in empty slots (i.e traffic intransit has precedence over traffic queued in the node).

The nodes also monitor the control slots in search of instances of their address in the destination fields of the control slot so as to receive the data and remove the payload (slot re-use is employed in this ring).

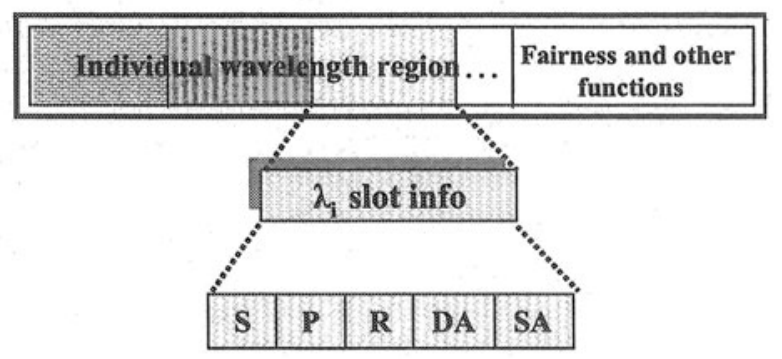

Figure 2. The control slot format

\section{A STRATEGY FOR QOS}

A focal point of DAVID is the support of services with demanding quality requirements and not just plain best-effort traffic. This introduces a significant challenge since the indiscriminate mixing of delay-sensitive and best-effort traffic results in uncontrollable loss for the former. This is caused by the elastic behaviour of the latter, which normally expands up to buffer overflow under the closed loop congestion control mechanisms of TCP. Though retransmissions rectify the loss for best-effort traffic, this is not possible for traffic with demanding QoS, which is also not generally amenable to modulation of its rate according to network conditions because of the intolerable delay of closed loop control message propagation. Thus delay-sensitive traffic is controlled preventively and must remain within rather predictable traffic profiles (i.e its temporal properties obey statistical limits which are exceeded with insignificantly low probability, say $10^{-9}$ ). Thus it has been ascertained by contract agreement (connection admission control-CAC) and source rate restrictions (policing) that traffic aggregations 
offered to the system by such a traffic class do not exceed the system capacity in its statistical fluctuations.

A study of CAC algorithms reveals that in order to guarantee proper performance to real-time services at the very low loss rate required, a very conservative allocation is needed and despite at times exhausting the system capacity, most of the time the system remains at low usage levels thus providing the opportunity of access to delay tolerant traffic which was waiting at the long entry buffers for an access opportunity [4]. If restrictions are placed to the total traffic accessing the system, then the minimum quality can be guaranteed for all (despite the fact that best-effort traffic does not need it) but the penalty is very low efficiency indeed.

The best strategy is to allow the mixing after making sure (through prioritised access) that best-effort traffic only expands when delay-sensitive traffic is at statistical low thus resulting in an opportune exploitation of bandwidth that would be otherwise wasted taking advantage of the fact that $\mathrm{BE}$ traffic is modulated by means of closed-loop congestion control. This approach is also recommended in [4]. Hence the value of access priorities in a system like DAVID where multiplexing is distributed. (In a centralised queuing point as inside switches/routers it is much easier to apply flow segregation and other complex scheduling policies achieving the same end result). Since policing units at system entry have ascertained no contract violations, there is confidence that the system capacity will not be exceeded and therefore access delay can remain within the design bounds. The throughputs for each node can vary according to rates agreed and paid for. This is assuming that an access priority mechanism is in operation guaranteeing that the presence of best-effort traffic will not present any competition to the delay sensitive traffic and it will be suppressed inside any available surplus bandwidth.

At moments of high statistical aggregations for the delay-sensitive traffic only a minimum bandwidth may remain available to best-effort traffic (or even zero depending on operator policy which in turn depends on charging policy). Normally the operator during provisioning and/or signalling may start not accepting delay-sensitive connections short of completely push out best-effort traffic. On the other hand it is conceivable to "sell" all the bandwidth to delay-sensitive connections leaving the best-effort to take advantage only of bandwidth left over from statistical fluctuations.

It is worth noting that there is no need to support a high number of MAC access priorities as is done in centralised queuing points in the adjacent $\mathbb{P}$ routers who are assumed to execute scheduling as well as drop policies of finer resolution. All is needed in the ring is to ascertain that the effects of scheduling will not be cancelled when going from the one router to the other through the shared-medium distributed multiplexer formed by the metro 
network. In line with the aggregation philosophy, it is enough to create two to four priority classes for groups of flows with similar characteristics. According to [5] just two classes of traffic aggregations (one for streamed and one for elastic traffic) are enough for QoS support. This claim is repeated in [6] albeit with some reservations. This MAC can however be extended to more priority classes at the penalty of small inefficiency for every additional priority level.

Before closing, it is worth commenting on the problem of fairness inherent in ring networks. The ring topology is prone to unfairness particularly under asymmetric traffic. The nodes sitting after destinations of heavy traffic have an advantage over other nodes since they get first the opportunity to see empty slots, fairness issues arise under asymmetric loads which necessitates an additional mechanism to distribute evenly the available bandwidth for best-effort traffic. Real-time traffic subject to acceptance control which includes resource reservation that guaranties that all demand will be satisfied. However best-effort traffic needs a separate fairness mechanism which is outside the scope of this work. Such a mechanism suitable for this case also is presented in [3] based on a variant of the SAT mechanism proposed for the meta-ring and other WDM rings.

\section{THE PRIORITY MECHANISM}

A novel priority mechanism different from those of the classical token ring featuring dynamic tracking of the fluctuations of the high-QoS traffic and suiting the metropolitan WDM ring will be presented in the sequence. This mechanism is described for segregation into two classes $A$ and $B$ enjoying respectively $1^{\text {st }}$ and $2^{\text {nd }}$ priority.

The concept employed for the priority mechanism is class reservation i.e. when no free slot is found, nodes mark busy slots for use exclusively by $1^{\text {st }}$ priority payloads (after they become free of course) using the $\mathrm{R}$ bit in the control channel. However this reservation is not meant for the use of the specific node which did the marking; but is at the disposal of any node with $1^{\text {st }}$ priority traffic. Since the marking is done on already busy slots it is likely that the slot will travel for part of a round trip (one half on average) and then be empty for another half round to reach the station that did the marking. But it is also possible that a station before the one who marked the slot may have $1^{\text {st }}$ priority traffic and use it. In this case however it has probably already marked another slot, which has reached the first node, which is also satisfied. So the rationale behind class reservations in a ring instead of the individual reservation in most other topologies [7] is efficiency. In general, when $1^{\text {st }}$ priority traffic increases, $1^{\text {st }}$ priority reserved slots proliferate and when it is 
reduced the reserved slots also are reduced. Note that there is no need to insert the address of the node that marks the slot since this is not an individual reservation. The algorithm mandates that, $1^{\text {st }}$ priority traffic will use an empty and marked slot in preference to an empty but unmarked slot to prevent a greedy behaviour over the $2^{\text {nd }}$ priority.

On the other hand when 1st priority traffic recedes, the markings need to be reduced in concert. Every node keeps track of its actions so that it will also remove an equal number of marks and therefore allow the sea of markings to $\mathrm{ebb}$ and flow according to 1 st priority traffic fluctuations. Nodes that after setting a reservation mark find an unmarked empty slot, they have to remove a reservation mark, thus the logic is that markings and unmarkings should tally in the long run while creating enough reservations in the interim to ferry high class slots to their destination ahead of other traffic. Regarding queue management, each node will maintain a separate queue per quality class. Upon arrival of each packet, it is inspected and classified according to its quality class based on information in its header, and then placed in the appropriate queue where automatically will receive the appropriate treatment by the MAC. FIFO order is enforced in each queue.

\section{PERFORMANCE ASSESSMENT}

To evaluate the performance of the mechanism a simulation model of the system was developed using the OPNET platform. The network model consisted of 16 wavelengths and 15 nodes. The system rate is $10 \mathrm{Gbps}$ per $\lambda$ (i.e. a total of $160 \mathrm{Gbps}$ ). Each node is fully equipped with 16 fixed receivers and 16 fixed transmitters so no contention for receivers or wavelengths exists. This choice was made so that the assessment of the priority algorithm is not complicated by other access contentions. For quick result collection the distance among the nodes is rather short, i.e. round trip time is $60 \mu \mathrm{s}$ and with a slot of a $1 \mu$ s length the ring holds only 66 slots per wavelength. The offered load is uniform with random distribution of destinations around the ring. The total load varies up to $200 \%$ of the total line rate. (With a truly random selection of destinations, i.e. mean distance equal to half a ring perimeter, the theoretical throughput is $200 \%$ of the ring rate since each slot carries on average two payloads to their destination per round). In practice the system remains stable for a bit more than $180 \%$ when loaded with Constant Bit Rate (CBR) traffic. With ON-OFF (Variable Bit Rate-VBR) sources instability starts at much lower levels depending on the source burstiness.

The most important simulation results are summarized in Table 1 where the mean delay is also shown and illustrated in the following Figures 3 to 6. 
Table 1. Simulation scenarios and results

\begin{tabular}{llllll}
\hline Scenario & Sources & $\begin{array}{l}\text { 1st priority } \\
\text { load }\end{array}$ & $\begin{array}{l}\text { 2nd priority } \\
\text { load }\end{array}$ & $\begin{array}{l}\text { 1st priority } \\
\text { mean delay }\end{array}$ & $\begin{array}{l}\text { 2nd priority } \\
\text { mean delay }\end{array}$ \\
\hline 1 & CBR & $180 \%$ & - & $1.25 \mu \mathrm{s}$ & - \\
2 & CBR & $70 \%$ & $70 \%$ & $0.55 \mu \mathrm{s}$ & $0.72 \mu \mathrm{s}$ \\
3 & $\mathrm{CBR}$ & $100 \%$ & $100 \%$ & $0.56 \mu \mathrm{s}$ & Unstable \\
4 & VBR & $60 \%$ & $60 \%$ & $0.74 \mu \mathrm{s}$ & $7.37 \mu \mathrm{s}$ \\
5 & VBR & $70 \%$ & $70 \%$ & $1.12 \mu \mathrm{s}$ & $26.9 \mu \mathrm{s}$ \\
6 & VBR & $80 \%$ & $80 \%$ & $1.95 \mu \mathrm{s}$ & Unbounded \\
7 & VBR & $90 \%$ & $90 \%$ & $2.41 \mu \mathrm{s}$ & Unbounded \\
8 & VBR & $100 \%$ & $100 \%$ & $3.4 \mu \mathrm{s}$ & Unbounded \\
\hline
\end{tabular}

The sources used in the first three scenarios produce uniformly distributed traffic with constant rates. In the first scenario there is only one traffic class. This is a benchmark to compare with the scenarios that use both priorities. In scenario 2 , both classes enjoy very good performance with $1^{\text {st }}$ priority (class A) exhibiting slightly lower access delay. The evolution of delay with time for some of the nodes is depicted in Figure 3. After some initial disturbance due to the sudden introduction of the load the two classes settle to a delay the values of $0.55 \mu$ s for class $A$ and $0.72 \mu$ s for class $B$ without appreciable difference among nodes.

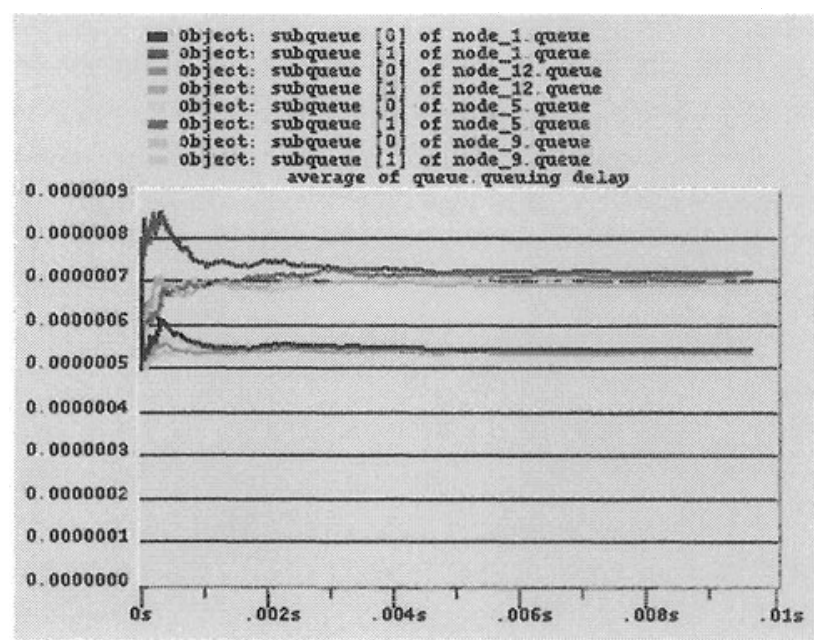

Figure 3. Scenario results

The value of the mechanism first becomes clear in scenario 3 (Figure 4), where the system seems unable to efficiently support the offered load of $200 \%$ which is above the total ring capacity. The action of the mechanism however allows the $1^{\text {st }}$ priority (class A) traffic to enjoy good performance 
leaving the $2^{\text {nd }}$ priority suffer the congestion. The delay of the $2^{\text {nd }}$ priority traffic (class B) increases constantly and almost linearly. It is remarkable that under such conditions, class A still enjoys the QoS necessary for realtime services. Traffic B queues grow indefinitely in the simulation model but in reality TCP closed loop mechanisms would detect losses and activate congestion avoidance. As a result rate will drop reflecting availability of bandwidth in the narrowest part of the loop (bottleneck). It is worth noticing the initial high delays due to the sudden jump of traffic generation from zero to $200 \%$, which causes some initial queuing, but this settles in about $1 \mathrm{~ms}$, as the mechanism jumps into action within $1 / 10^{\text {th }}$ of this time i.e., in about $100 \mu \mathrm{s}$ which is just 2-3 round trips. The dynamic behaviour of the mechanism will be investigated in more detail in the last scenario below.

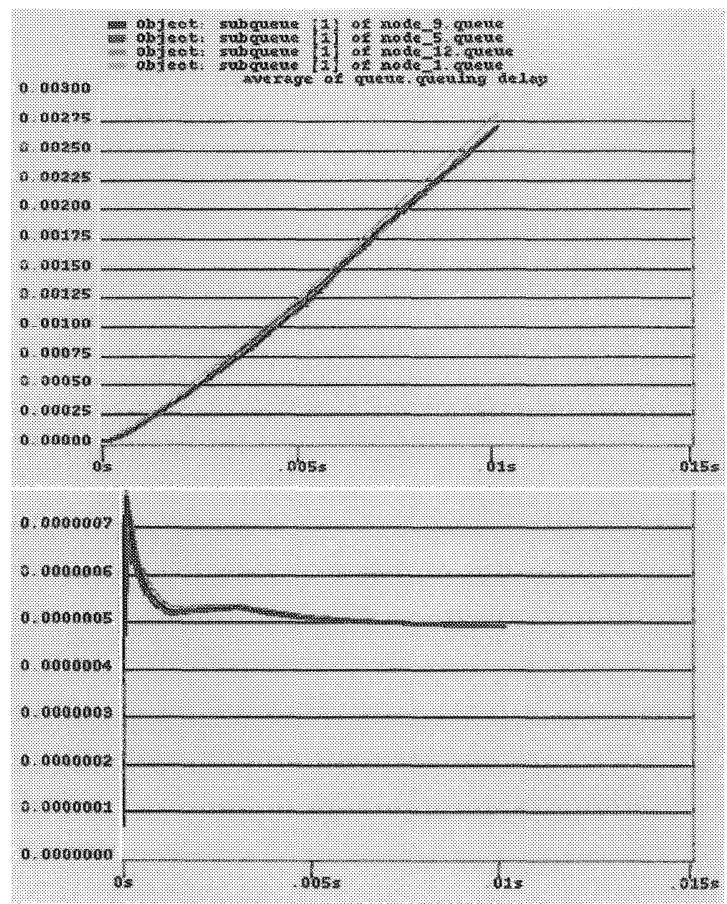

Figure 4. Scenario 3 results

In the runs shown in scenarios 4 to 8 , the system was uniformly loaded with variable sources of the ON-OFF type. The duration of the ON and OFF periods was geometrically distributed while a fixed inter-arrival time was employed during the $\mathrm{ON}$ period at a peak rate which was 2.5 times the average rate for class A traffic and 6 times for class B (i.e., a burstiness of 2.5 and 6 respectively). The load was equally divided among class $A$ and the 
class $\mathrm{B}$. The run duration was chosen for a confidence interval of $8 \%$. The mean rate varied to produce the total offered load as shown in the table (expressed as a percentage of the line rate) resulting in the corresponding mean delay values. The curve of the delay as a function of the total offered load is depicted in figure with class B shown on the top and class A on the bottom). Note that again in all cases Class A enjoys good performance as necessary to satisfy its demanding QoS. Class B is serviced with good performance (albeit not as good as that of class A) while the load is within capacity. Of course when the total load can not be supported by the system the delays are shown unbounded but as explained below this is due to the fact that TCP action is not modeled by the sources. It is worth noting that although with CBR sources the system could support at a total load of $180 \%$, with the used ON-OFF sources it is already unstable at $160 \%$. With increased source burstiness the stability limit is reached at an even lower loading. This of course a general result for any multiplexer and by no means restricted to the system under study.
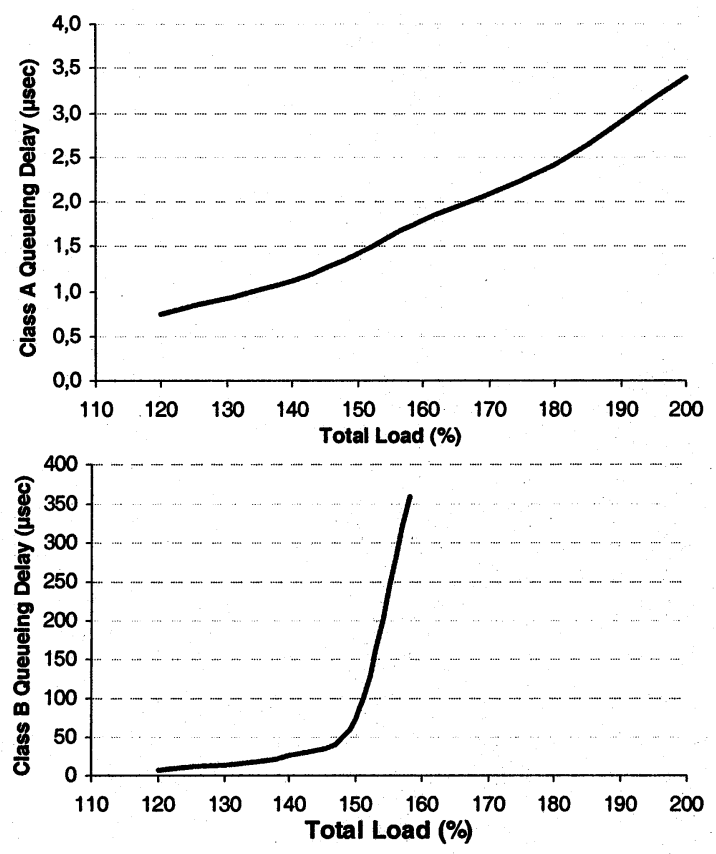

Figure 5. Delay vs load for ON-OFF sources (scenario 4-8)

To investigate the dynamic behaviour of the mechanism sudden changes were introduced in the offered load of class $A$ in the final scenario 9 (not shown in table). Ideally the number of slots marked for class A should 
increase as class A traffic increases suppressing the service of class B packets but retract fast if class A traffic suddenly drops leaving room for servicing more class $\mathrm{B}$ packets. To test this a simulation was run in which the ring is initially loaded at $180 \%$ with $100 \%$ consisting of class A traffic and $80 \%$ class $\mathrm{B}$ using fixed rate sources uniformly distributed. The results are shown in Figure 4 where the evolution of packet delay with time is shown for both classes. Class A traffic (bottom figure) stays unaware of the high loading, while $2^{\text {nd }}$ priority class $B$ queues start to grow in an unstable manner. It is worth noting the initial temporary peak of the delay of class A due to the sudden jump of traffic generation from zero to $200 \%$, but quickly (in about $1 \mathrm{~ms}$ ) queues settle as the mechanism jumps into action.

At about $3 \mathrm{~ms}$ the $1^{\text {st }}$ priority load drops suddenly from $100 \%$ down to $50 \%$. Class A traffic experiences a marginal improvement in access delay, while class B queues respond immediately and their size starts to drop. The released capacity is made immediately available to class $B$ with a corresponding increase in throughput, which is reflected on the queue size curve which bends down and starts decreasing.

The load of class A goes back to $100 \%$ again $3 \mathrm{~ms}$ later and again the mechanism immediately creates priority slots which shield class $\mathrm{A}$. The mechanism follows the load fluctuations returning fast any released bandwidth to free access use by class B and vice-versa. Please note that the vertical axis scale is different for the two priorities since the maximum values of delay are much higher for class $B$. 


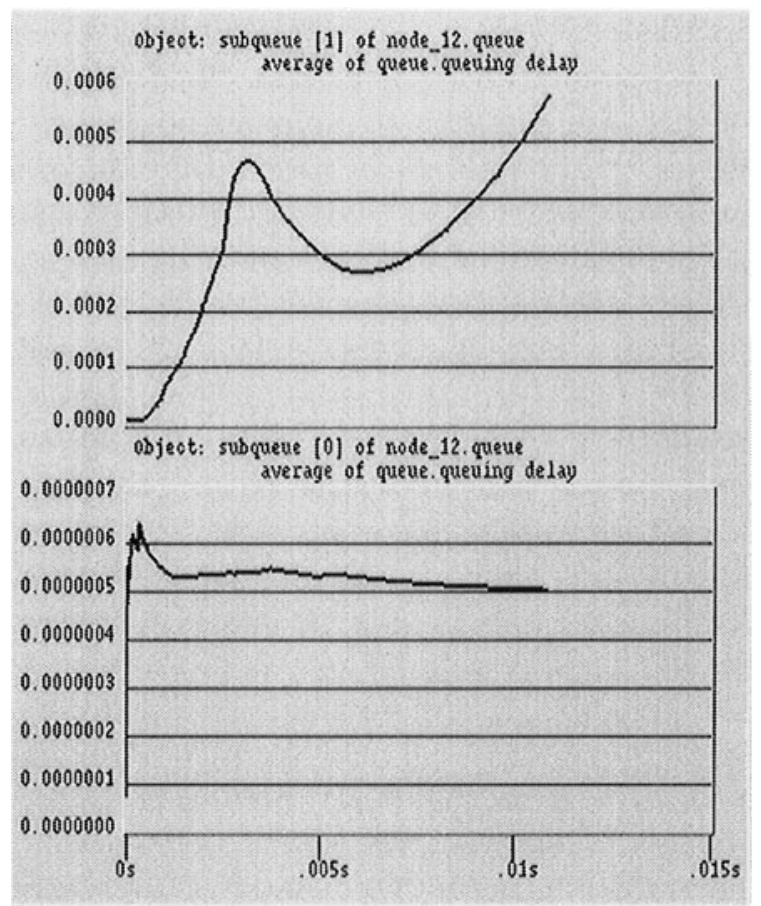

Figure 6. Dynamic behavior of the mechanism

\section{CONCLUSIONS}

The services that will fill the high bandwidth pipes created by revolutionary developments in optical networking will largely consist of real-time video and audio based traffic with demanding quality requirements necessitating systems able to support such requirements beyond plain besteffort traffic. WDM-based metropolitan rings will play a key role in concentrating traffic towards the fat pipes of the core network. Support of QoS-demanding services will be crucial in filling the pipes since most of the traffic will be real-time video/audio. The MAC protocol arbitrating the access to such rings requires a mechanism to support the segregation of quality demanding traffic from best-effort traffic. The presented mechanism presents satisfactory performance with fast adaptation to a changing traffic mix, which allows for high efficiency. The whole effect is a system that satisfies at once quality constraints for real-time traffic and a high utilisation based-on the delay tolerance and closed-loop control of best-effort traffic that allows it to wait in buffers for an opportunity to enter or be dropped and retransmitted whenever traffic aggregates exceed capacity. 


\section{ACKNOWLEDGMENTS}

The work presented in this paper was partially funded by the EU project IST-1999-11742 "DAVID".

\section{REFERENCES}

[1] A. Stavdas, C. Skoufis, I. Angelopoulos, G. Stassinopoulos, I. Pountourakis, "On Multi- $\lambda$ Packet Labeling for Metropolitan and Wide-Area Optical Networks". Photonic network communications Journal, Vol.3, No.1, January 2001.

[2]D. Chiaroni et al, "Towards $10 \mathrm{~Tb} / \mathrm{s}$ optical packet routers for the backbone" $26^{\text {th }}$ European Conference on Optical Communications, Vol.4 pp.73-74, Munich, September 3. 7,2000

[3] M.A. Marsan, A. Bianco, E. Leonardi, A. Morabito, F. Neri "All optical WDM Multi-rings with Differentiated QoS", IEEE Comm. Mag. Feb. 1999, Vol. 37, No. 2, pp 58-66.

[4] Jim Roberts, "Traffic Theory and the Internet", IEEE Comm. Mag. Jan 2001, Vol 39, No. 1 pp 94-99.

[5] Lindberger K., "Balancing QoS, Pricing and Utilisation in multi-service Networks with Stream and Elastic Traffic", in Proceeding of the ITC, 16 June 1999, pp. 1127-36.

[6] Klaus Dolzer, Wolfgang Payer, Markus Eberspacher, "A simulation study on traffic aggregation in multi-service networks", Proceedings of the IEEE Conference on High Performance Switching and Routing, 26-29 June 2000, Heidelberg Germany

[7] J. D. Angelopoulos, N. I. Lepidas, E. K. Fragoulopoulos, I.S. Venieris, ${ }^{66}{ }^{6}$ DMA multiplexing of ATM cells in a residential access SuperPON", IEEE Journal on Selected Areas in Communications, Special issue on high capacity optical transport networks, Vol. 16, No. 7, September, 1998. 